\title{
Atomic Structure Affects the Directional Dependence of Friction
}

\author{
A. J. Weymouth, ${ }^{1, *}$ D. Meuer, ${ }^{1}$ P. Mutombo, ${ }^{2}$ T. Wutscher, ${ }^{1}$ M. Ondracek, ${ }^{2}$ P. Jelinek, ${ }^{2}$ and F. J. Giessibl ${ }^{1}$ \\ ${ }^{1}$ Institute of Experimental and Applied Physics, University of Regensburg, D-93053 Regensburg, Germany \\ ${ }^{2}$ Institute of Physics, Academy of Sciences of the Czech Republic, Cukrovarnicka 10, 16200 Prague, Czech Republic
}

(Received 26 April 2013; published 18 September 2013)

\begin{abstract}
Friction between two objects can be understood by the making, stretching, and breaking of thousands of atomic-scale asperities. We have probed single atoms in a nonisotropic surface [the H-terminated $\mathrm{Si}(100)$ surface] with a lateral force microscope operating in noncontact mode. We show that these forces are measurably different, depending upon the direction. Experimentally, these differences are observable in both the line profiles and the maximum stiffnesses. Density functional theory calculations show a concerted motion of the whole Si dimer during the tip-sample interaction. These results demonstrate that on an asperity-by-asperity basis, the surface atomic structure plays a strong role in the directional dependence of friction.
\end{abstract}

DOI: 10.1103/PhysRevLett.111.126103

PACS numbers: 68.35.Af, 07.79.Lh, 07.79.Sp

Any measurement of friction requires an understanding of the contact between the two surfaces. When measuring friction at the nanoscale, a common method is friction force microscopy (FFM), in which the coefficient of kinetic friction is determined by measuring the energy loss as a tip slides over an atomically flat surface [1]. It has been invaluable in investigating friction as a function of crystal face [2], electronic structure [3], and of direction of motion $[4,5]$. However, many systems studied with FFM can be difficult to interpret because of the large number of atoms involved in the multiple asperities between tip and sample. For example, while Liley et al. were able to relate friction anisotropy to the tilt of a molecular surface layer, they were not able to explain the observed friction asymmetry [6]. Even in the case of a well-understood molecular surface, Fessler et al. observed a breakdown of the commonly used Prandtl-Tomlinson model [7]. In our work, we use a lateral force microscope (LFM) operating in noncontact mode and measure tip-sample interaction with a single-atom asperity [8]. While LFM has observed atomic interactions [9-11], dependence on crystallographic direction has not been reported. By combining our theoretical and experimental results, we are able to understand anisotropic nanotribological interactions at the atomic level. Specifically, we have investigated motion in two high-symmetry directions over a H-terminated Si dimer.

In FFM, a cantilever is depressed onto a surface and dragged along it, with the torsional bending of the cantilever used to measure the lateral forces $[12,13]$. In special cases, the tip-sample interaction can be very well characterized, such as in an investigation by Dienwiebel et al., who probed graphene on graphite and observed superlubricity in certain directions of motion [14]. Normal noncontact atomic force microscopy, in which an atomically sharp tip is oscillated perpendicular to the surface, can also be used to probe lateral forces, albeit indirectly [15]. This has the disadvantage that a full three-dimensional data set must be acquired. A more direct measure of this lateral force gradient is to excite the torsional mode of the cantilever such that the tip oscillates laterally across the surface, exploiting the benefits of noncontact atomic force microscopy in a lateral geometry $[9,10,16,17]$. Here, we have chosen a different option. We have constructed a sensor, as shown in Fig. 1(a), such that the tip oscillates parallel to the sample surface at the fundamental mode of the cantilever. This technique allows a single-atom asperity to directly probe the lateral stiffness convoluted over the oscillation of the tip [11].

In this Letter, we describe LFM measurements showing anisotropy on the atomic level. While STM measurements of adsorbates have previously reported surface friction anisotropy on the atomic scale $[18,19]$, they did not measure lateral forces. We have carefully chosen a system that allows us to probe two high-symmetry directions above the surface without requiring coarse motion or rotation of the sample. Because we can collect data of two high-symmetry directions with the tip oscillating in the same direction within one image, we can be certain that these differences in the data are due to anisotropy of the sample. (See the Supplemental Material [20] for further details.) Furthermore, a single-atom asperity allows us to fully model the system from first principles. The strong agreement between the measured and calculated lateral forces indicates that we are accurately modeling the experimental setup, and can confidently describe the mechanism behind this anisotropy.

The $\mathrm{Si}(100)$ surface reconstructs into rows of Si dimers that align on each atomic terrace but are orthogonal to the rows on the terrace one step down. When unsaturated, the Si dimers are highly reactive and buckle rapidly in a rocking motion at room temperature. By saturating them with hydrogen, they assume a $2 \times 1$ symmetric configuration and are much less reactive to adsorbates [21]. If we align the sample such that the tip oscillation is parallel to 
(a)

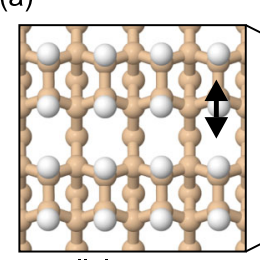

parallel

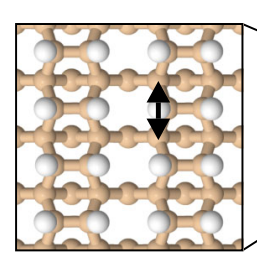

perpendicular
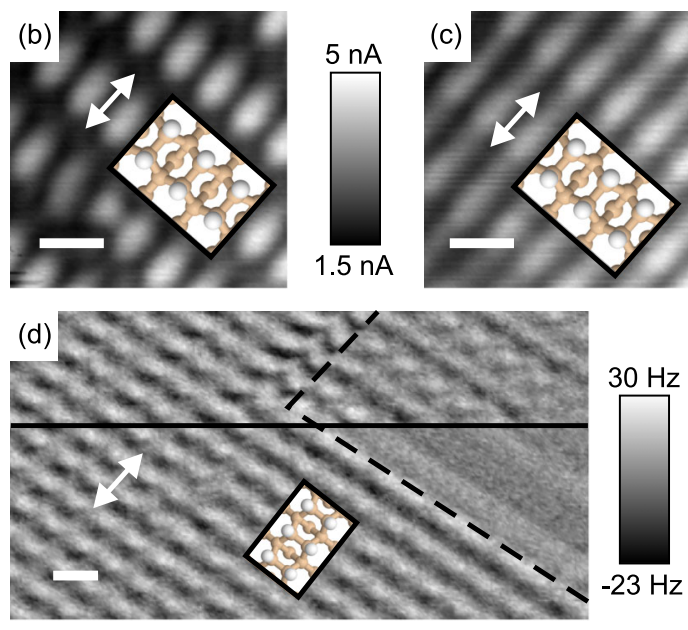

FIG. 1 (color online). (a) A schematic of the experimental setup. By cutting the $\mathrm{Si}$ wafer on the $\{011\}$ planes, the (011) crystallographic direction can be aligned with the tip oscillation. By moving from one terrace to another, data can be acquired with the tip oscillating either parallel or perpendicular to the $\mathrm{Si}$ dimers. (b) Constant-height $I_{t}$ data at a lateral oscillation amplitude of $50 \mathrm{pm}$. (c) Same as (b) but with an amplitude of $300 \mathrm{pm}$. (d) $\Delta f$ data over two terraces, with the step edge highlighted with a dashed line. A bias of $1.5 \mathrm{~V}$ was applied. Above the black line, $I$ is used to control the tip height (set point of $4 \mathrm{nA}$ ). Below it, the feedback is switched off. The tip oscillation in all subfigures is indicated by double-headed arrows. Images are taken with a scan angle of $45^{\circ}$. White scale bars represent $500 \mathrm{pm}$.

the Si dimers on a given terrace, then by moving to the lower (or upper) terrace, we can acquire data while the tip oscillates perpendicular to the Si dimers, as shown in Fig. 1(a). This allows us to investigate the stiffness both parallel and perpendicular to the Si dimers. By cutting our Si wafer on the $\{011\}$ planes, the (011) direction can be aligned with the tip oscillation.

As a first investigation, we collected constant-height STM data at both small and large oscillations, shown in Figs. 1(b) and 1(c). The high-symmetry crystal directions are labeled in Fig. 1(a). Comparing Figs. 1(b) and 1(c), it is clear that the increased amplitude leads to a blurring in a direction parallel to the dimers and that the tip is indeed oscillating along this high-symmetry direction.

To probe two different terraces at room temperature, acquiring data without feedback is not feasible. Furthermore, the frequency shift $\Delta f$ is not monotonic over the surface [as shown in Fig. 1(d)], which means that it cannot be used to control the tip-sample height. We therefore collect data using the tunneling current $I$ to control the tip height. Figure 1(d) shows data collected with the feedback on and off. These data are taken over a step edge, marked as a dashed line. For the part of the image above the black line, the feedback was on and contrast on both terraces can be seen. With the feedback off, below the solid line, the contrast on the lower terrace is greatly reduced, whereas the contrast on the upper terrace does not change significantly. Therefore, the tip motion due to the STM topography has a negligible influence on the $\Delta f$ data as long as the tip stays on a given terrace.

Figure 2(a) shows $\Delta f$ data that were collected over two terraces. The dashed line indicates the step edge. The $\Delta f$ contrast is greater when the tip oscillates perpendicular to the Si dimers, which we observed over a range of current set points, shown in Supplemental Fig. 1. From this image, we extracted a line profile from each terrace, shown in Figs. 2(b) and 2(c), where the data are marked in black dots and the measurement uncertainty is smaller than the markers themselves. (See the Supplemental Material [20] for further details.) Given the clear periodicity of the data, and in order to facilitate the analysis, we fit each line profile with a Fourier series. The appeal of fitting this data with a Fourier series is that the deconvolution of the tip oscillation can be performed analytically and that noise is reduced in the first processing step. $\Delta f$ is proportional to the tip-sample stiffness $k_{\mathrm{ts}}$ convolved over the tip motion as it oscillates. In this case, the deconvolved stiffness is also a Fourier series, with the same periodicity and phase, where the coefficients are related via a Bessel function of the first order. (See the Supplemental Material [20] for details.)

The profiles of the deconvoluted lateral stiffnesses $k_{\mathrm{ts}}$ are shown in Figs. 2(b) and 2(c). In motion perpendicular to the dimers, a maximum contrast of $8.42 \pm 0.13 \mathrm{~N} / \mathrm{m}$ is measured, whereas parallel to the $\mathrm{Si}$ dimers it is $7.29 \pm 0.13 \mathrm{~N} / \mathrm{m}$, shown in Supplemental Fig. 2. By assuming that the highest force gradient occurs where the lateral force is zero, the deconvolved lateral stiffnesses can be integrated to yield lateral force, shown by solid black lines in Figs. 2(b) and 2(c).

To further explore these observations, we performed first-principles calculations with the FIREBALL density functional theory code [22]. Although we used a bulk W tip, it was prepared with standard STM techniques, including strong surface pokes and bias pulses, which tend to form small $\mathrm{Si}$ clusters on the tip apex. Therefore, we used a well-tested $\mathrm{Si}$ dimer tip model 

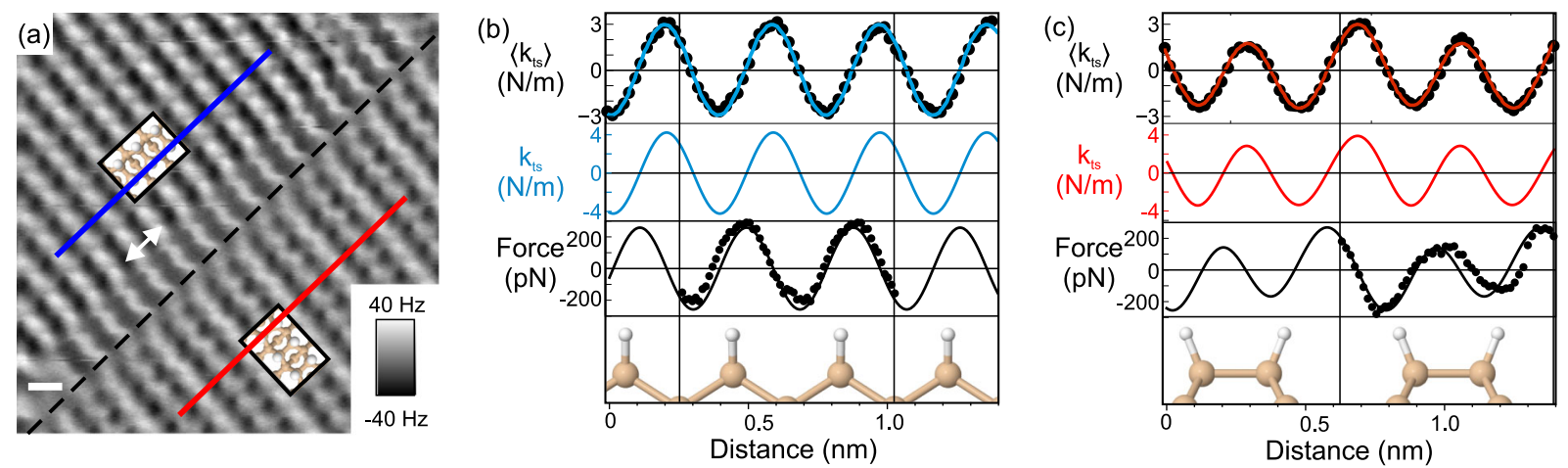

FIG. 2 (color online). (a) $\Delta f$ data taken over two terraces (step edge is highlighted by a dashed line). Scan angle $45^{\circ}$, oscillation amplitude $100 \mathrm{pm}$, bias voltage $1.5 \mathrm{~V}$, set point $3.4 \mathrm{nA}$. The white scale bar represents $500 \mathrm{pm}$. Line profiles were taken along the solid lines shown in (a). The direction of the tip oscillation is shown as a white arrow. (b) Convoluted force gradient for the line profile on the upper left terrace, corresponding to the tip oscillation perpendicular to the Si dimers. From the top down: Data are black dots, and the Fourier fit is a solid line; deconvolved force gradient from the Fourier fit; lateral force as evaluated from the data (solid line) and calculated from density functional theory (black points); structural model. (c) As in (b) for the lower-right terrace.

[23] to mimic the experimental tip apex. The solid dots in Figs. 2(b) and 2(c) show the calculated lateral forces at a vertical distance of $170 \mathrm{pm}$ from the surface $\mathrm{H}$ atoms to the apex atom of the tip. Not only are the profiles in good agreement, but the magnitudes of the forces are also in excellent agreement. At first, it might seem unclear why the tip is not between the two dimers at the point of the greatest force gradient, but the dimer tip does not possess lateral symmetry, as can be seen in Fig. 3. In other words, the lateral position of the atomic core is not necessarily the location with the strongest force interaction between tip and sample.

We also investigated a rotated $\mathrm{Si}$ dimer tip, as shown in Supplemental Fig. 3. Both these data sets confirmed our previous assumption that where the force gradient is the largest, the lateral force is zero. They both also clearly show a difference in the force profile in the two directions of motion.

In order to further understand the tip-sample interaction, we can examine the atomic displacements as the Si dimer
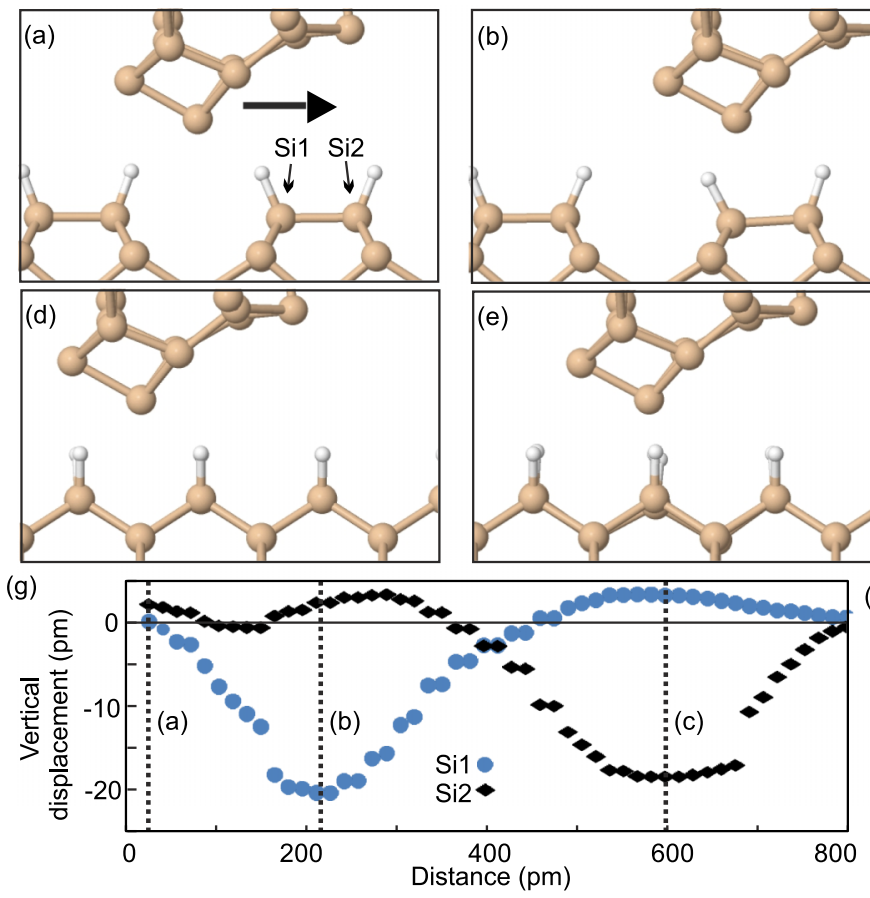

$(e)$

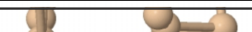

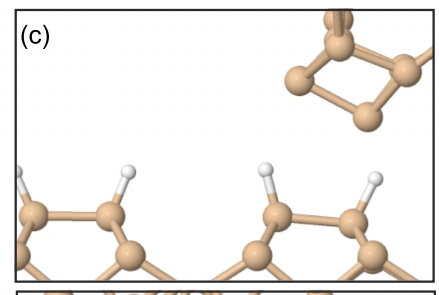
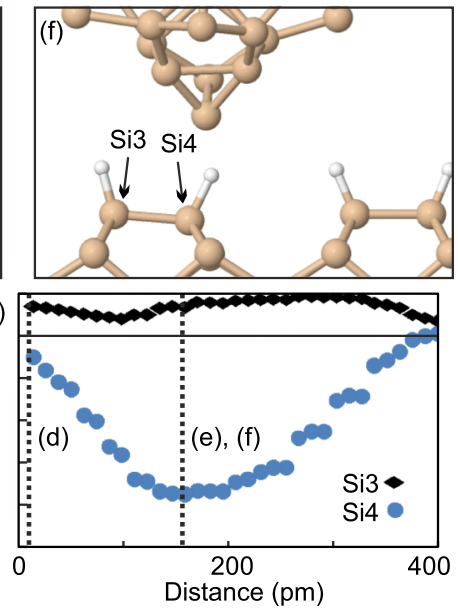

FIG. 3 (color online). (a)-(c) Relaxed atomic positions corresponding to motion parallel to the Si dimer, indicated by the dark arrow in (a), for various positions over a Si dimer as indicated by the vertical dashed lines in (g). (d)-(f) Motion perpendicular to the dimer, indicated by the dark arrow in (d), with positions indicated by the dashed lines in (h). (e),(f) At the same tip position but rotated to show the dimer displacement. $(\mathrm{g}),(\mathrm{h})$ Shown is the vertical displacement of the $\mathrm{Si}$ atoms of the dimer below the tip. 
tip moves across the surface. As it does, the $\mathrm{H}$ atoms displace away, indicating that we are in the repulsive regime. Figures 3(a)-3(c) show the atomic displacement as the tip moves across the surface parallel to the Si dimer. Figures 3(b) and 3(c) were chosen because they show the greatest deflection of the Si dimer. Figure 3(g) shows the calculated displacement of the two Si dimer atoms in the $z$ direction, as the tip moves across the dimer. Supplemental Figure 4 shows the displacements in the plane of the surface. From Fig. 3(g), it can be seen that the dimer responds to the tip in a rocking motion, with one $\mathrm{Si}$ atom moving down and the other simultaneously moving up.

In Figs. 3(d) and 3(e), tip motion perpendicular to the Si dimer is considered. Figure 3(e) shows the tip location at which the $\mathrm{H}$ atom deflects furthest. If we inspect the deflection at this point in the plane of the Si dimer, shown in Fig. 3(f), then we again see the Si dimer deflecting in a rocking motion. This can also be seen in Fig. 3(h) by the vertical deflection of the $\mathrm{Si}$ atoms. Supplemental Figure 5 shows the atomic displacements in the plane of the surface.

The H-terminated $\mathrm{Si}(100)$ surface has been previously used to investigate friction by Cannara et al. [24]. In this work, friction force microscopy was used to compare the energy dissipation over $\mathrm{H}$ - and D-terminated (deuteriumterminated) surfaces. Greater energy dissipation was observed on the H-terminated surface and attributed to the higher phonon frequency. The energy dissipation was a combination of energy lost to the substrate and momentum transferred back to the tip. While it would be difficult to observe similar differences between $\mathrm{H}$ and $\mathrm{D}$ in lateral stiffnesses, given the similarity in their bond stiffnesses, the energy dissipation (probed by the drive signal) might show a difference.

We have presented data showing clear differences in lateral stiffness along two high-symmetry directions and have been able to successfully reproduce these data with a model involving a realistic tip. With our model, we showed that the Si dimer responds in a rocking motion. The experimental setup was carefully chosen such that differences between the two terraces would only be observed if the lateral stiffness of a $\mathrm{Si}$ dimer was not the same in parallel and perpendicular directions. Crystallographic and dimer alignment does play a measurable role on the welldefined $\operatorname{Si}(100)$ surface with respect to frictional forces. More generally, our findings highlight the importance of the surface structure and bonding symmetry on the directional dependence of static friction at the atomic and molecular level.

A. J.W., D. M., T. W., and F. J.G. kindly acknowledge financial support from the Deutsche Forschungsgemeinschaft (Grant No. GRK 1570). M. O. was supported by the Czech Science Foundation (GAČR), Project No. P204/11/P578. M. O., P. M. and P. J. acknowledge financial support from GAAV Project No. M100101207.
*Corresponding author.

jay.weymouth@physik.uni-regensburg.de

[1] C. M. Mate, G. M. McClelland, R. Erlandsson, and S. Chiang, Phys. Rev. Lett. 59, 1942 (1987).

[2] G. Gao, R. J. Cannara, R. W. Carpick, and J. A. Harrison, Langmuir 23, 5394 (2007).

[3] J. Y. Park, D. F. Ogletree, P. A. Thiel, and M. Salmeron, Science 313, 186 (2006).

[4] S. Fujisawa, E. Kishi, Y. Sugawara, and S. Morita, Phys. Rev. B 51, 7849 (1995).

[5] R. M. Overney, H. Takano, M. Fujihira, W. Paulus, and H. Ringsdorf, Phys. Rev. Lett. 72, 3546 (1994).

[6] M. Liley, D. Gourdon, D. Stamou, U. Meseth, T. M. Fischer, C. Lautz, H. Stahlberg, H. Vogel, N. A. Burnham, and C. Duschl, Science 280, 273 (1998).

[7] G. Fessler, I. Zimmermann, T. Glatzel, E. Gnecco, P. Steiner, R. Roth, T. D. Keene, S.-X. Liu, S. Decurtins, and E. Meyer, Appl. Phys. Lett. 98, 083119 (2011).

[8] O. Pfeiffer, R. Bennewitz, A. Baratoff, E. Meyer, and P. Grütter, Phys. Rev. B 65, 161403(R) (2002).

[9] S. Kawai, N. Sasaki, and H. Kawakatsu, Phys. Rev. B 79, 195412 (2009).

[10] S. Kawai, T. Glatzel, S. Koch, B. Such, A. Baratoff, and E. Meyer, Phys. Rev. B 81, 085420 (2010).

[11] F. J. Giessibl, M. Herz, and J. Mannhart, Proc. Natl. Acad. Sci. U.S.A. 99, 12006 (2002).

[12] G. Meyer and N. M. Amer, Appl. Phys. Lett. 57, 2089 (1990).

[13] O. Marti, J. Colchero, and J. Mlynek, Nanotechnology 1, 141 (1990).

[14] M. Dienwiebel, G. S. Verhoeven, N. Pradeep, J. W. M. Frenken, J. A. Heimberg, and H. W. Zandbergen, Phys. Rev. Lett. 92, 126101 (2004).

[15] B. J. Albers, T.C. Schwendemann, M.Z. Baykara, N. Pilet, M. Liebmann, E. I. Altman, and U.D. Schwarz, Nat. Nanotechnol. 4, 307 (2009).

[16] S. Kawai, T. Glatzel, S. Koch, B. Such, A. Baratoff, and E. Meyer, Phys. Rev. Lett. 103, 220801 (2009).

[17] S. Kawai, S.-i. Kitamura, D. Kobayashi, and H. Kawakatsu, Appl. Phys. Lett. 87, 173105 (2005).

[18] S.-W. Hla, K.-F. Braun, and K.-H. Rieder, Phys. Rev. B 67, 201402(R) (2003).

[19] J. A. Stroscio and R. J. Celotta, Science 306, 242 (2004).

[20] See Supplemental Material at http://link.aps.org/ supplemental/10.1103/PhysRevLett.111.126103 for Supplemental Methods and Figures.

[21] J. J. Boland, Phys. Rev. Lett. 65, 3325 (1990).

[22] J. P. Lewis, P. Jelínek, J. Ortega, A. A. Demkov, D. G. Trabada, B. Haycock, H. Wang, G. Adams, J. K. Tomfohr, E. Abad, H. Wang, and D. A. Drabold, Phys. Status Solidi B 248, 1989 (2011).

[23] P. Pou, S. A. Ghasemi, P. Jelínek, T. Lenosky, S. Goedecker, and R. Perez, Nanotechnology 20, 264015 (2009).

[24] R. J. Cannara, M. J. Brukman, K. Cimatu, A. V. Sumant, S. Baldelli, and R.W. Carpick, Science 318, 780 (2007). 